\title{
Familial amyloid precursor protein mutants cause caspase-6-dependent but amyloid $\beta$-peptide-independent neuronal degeneration in primary human neuron cultures.
}

\author{
SN Sivananthan ${ }^{1,2}$, AW Lee ${ }^{1,2}$, CG Goodyer ${ }^{3}$ and AC LeBlanc ${ }^{\star, 1,2}$
}

Although familial Alzheimer disease (AD)-associated autosomal dominant mutants have been extensively studied, little is known about the underlying molecular mechanisms of neurodegeneration induced by these mutants in AD. Wild-type, Swedish or London amyloid precursor protein (APP) transfection in primary human neurons induced neuritic beading, in which several co-expressed proteins, such as enhanced green fluorescent protein, red fluorescent protein (RFP)-tau and RFP-ubiquitin, accumulated. APP-induced neuritic beading was dependent on caspase- 6 (Casp6), because it was inhibited with $5 \mu$ M z-VEID-fmk or with dominant-negative Casp6. Neuritic beading was independent from APP-mediated amyloid $\beta$-peptide (A $\beta)$ production, because the APPM596V (APPMV ) mutant, which cannot generate A $\beta$, still induced Casp6-dependent neuritic beading. However, the beaded neurons underwent Casp6- and $A \beta$-dependent cell death. These results indicate that overexpression of wild-type or mutant APP causes Casp6-dependent but A $\beta$-independent neuritic degeneration in human neurons. Because Casp 6 is activated early in $A D$ and is involved in axonal degeneration, these results suggest that the inhibition of Casp6 may represent an efficient early intervention against familial forms of $A D$. Furthermore, these results indicate that removing $A \beta$ without inhibiting Casp6 may have little effect in preventing the progressive dementia associated with sporadic or familial $A D$.

Cell Death and Disease (2010) 1, e100; doi:10.1038/cddis.2010.74; published online 18 November 2010

Subject Category: Neuroscience

Strong activity of caspase-6 (Casp6) determined by immunohistochemistry or immunoprecipitation, with neoepitope antisera to the active p20 subunit of Casp6 and to tau (microtubule-associated protein tau) or $\alpha$-tubulin (Tub) cleaved by Casp6 (tau $\Delta$ Casp6, tau cleaved by Casp6; or Tub $\Delta$ Casp6, tubulin cleaved by Casp6), is present in the hippocampus and affected cortices of sporadic and familial forms of Alzheimer disease (AD). ${ }^{1-4}$ Casp6 activity is present in the neuropil threads, neurofibrillary tangles and neuritic senile plaques of the brain of mild cognitively impaired and mild-to-severe AD individuals. ${ }^{2}$ Casp6 is also activated in the entorhinal cortex of non-cognitively impaired aged individuals that nevertheless had the lowest global cognitive scores of the group. ${ }^{2}$ As the entorhinal cortex is the area first affected in $A D$ brains, these findings suggest that Casp6 could be an early instigator of $A D$. In contrast to the other two short pro-domain effector caspases (Casps), caspase-3 (Casp3) and caspase-7 (Casp7), Casp6 has not been well investigated.

The high levels of active Casp6 in familial AD brain ${ }^{1}$ incite the question of whether familial AD-associated mutant proteins induce Casp6 activation and if this activation depends on $\mathrm{A} \beta$. Familial AD occurs either via APP duplication or single point mutations of the amyloid precursor protein (APP) and Presenilin genes. ${ }^{5,6}$ No studies have reported the effect of familial AD-associated mutant proteins on Casp6 activity. However, expression of the APP V717I London (Lo) mutant induces $\mathrm{A} \beta$-mediated Casp-dependent condensed chromatin in primary cortical neurons, ${ }^{7}$ and expression of the APP Lo mutation induces DNA fragmentation in COS or COSlike cell lines. ${ }^{8,9}$ Condensed chromatin and DNA fragmentation are both indicative of apoptotic cell death but the exact Casp responsible was not clearly identified in these studies. Expression of the APP Swedish (Sw) mutation increases the vulnerability of transfected PC12 cells to oxidative stress and the cells undergo Casp3-dependent cell death. ${ }^{10}$ Because familial mutants increase amyloid $\beta$-peptide $(\mathrm{A} \beta)$ production, $\mathrm{A} \beta$ is generally thought to cause $\mathrm{AD}$ by synaptic or neuronal toxicity (reviewed in Selkoe and Podlisny ${ }^{11}$ ). However, it is also possible that yet undiscovered natural functions are disrupted in mutant genes associated with familial AD, and cause undue stress to neurons before or independently from $\mathrm{A} \beta$ production. Indeed, cellular stresses induce overproduction of $\mathrm{A} \beta$ in a Casp-dependent manner, ${ }^{12,13}$ thus placing $\mathrm{A} \beta$ downstream of an initial stress.

\footnotetext{
${ }^{1}$ Bloomfield Center for Research in Aging, Lady Davis Institute for Medical Research, Jewish General Hospital, 3755 Chemin Côte Ste-Catherine, Montreal, Quebec, Canada; ${ }^{2}$ Department of Neurology and Neurosurgery, McGill University, 3775 University Street, Montreal, Quebec, Canada and ${ }^{3}$ Department of Pediatrics, McGill University, Montreal, Quebec, Canada

${ }^{*}$ Corresponding author: A LeBlanc, Bloomfield Center for Research in Aging, Lady Davis Institute for Medical Research, Sir Mortimer B Davis Jewish General Hospital, 3755 ch. Côte Ste-Catherine, Montréal, QC, Canada H3T 1E2. Tel: + 1 (514) 340 8260; Fax: + 1(514) 340 8295; E-mail: andrea.leblanc@mcgill.ca

Keywords: amyloid precursor protein; caspase-6; neurodegeneration; amyloid $\beta$-peptide.

Abbreviations: APP, amyloid precursor protein; AD, Alzheimer disease; tau, microtubule-associated protein tau; Sw, Swedish; Lo, London; EGFP, enhanced green fluorescent protein; RFP, red fluorescent protein; Tub, tubulin; $A \beta$, amyloid $\beta$-peptide; Casp, caspase

Received 22.6.10; accepted 10.9.10; Edited by A Verkhrasky
} 
Pro-Casp-6 (Casp6) is a cytosolic cysteinyl protease zymogen that is activated by self- or exo-proteolytic processing into a p20 and p10 subunit that reassemble to form the hetero-tetrameric active Casp6. ${ }^{14,15}$ Casp6 has often been placed downstream of Casp3 and Casp7 in the apoptotic cascade, as it is proteolytically processed and activated by these two enzymes. ${ }^{16}$ However, Casp6 is active in the absence of Casp3 and Casp7 in serum-deprived human primary neuronal cultures, and Casp6 activity precedes effector or initiator Casp activation in several cell types. ${ }^{13,17-19}$ Unlike the other effector Casps, active Casp6 is not always associated with apoptotic cell death. In AD, active Casp6positive neurons do not show apoptotic morphology. ${ }^{3}$ Microinjection of active Casp6, but not Casp3 or Casp7, induces a protracted type of cell death in human neurons. ${ }^{20}$ Similarly, activation of Casp6 in HEK293T cells does not induce cell death detectable by MTT reduction, sub- $\mathrm{G}_{1}$ content assessed by FACS analyses or Casp3 activation. ${ }^{15}$

Recently, Casp6 has been recognized as highly important in inducing axonal degeneration, whereas Casp3 induces cell death in APP-mediated death receptor 6 signaling in trophicfactor-deprived commissural, sensory and motor mouse neurons. ${ }^{21}$ Although active Casp6 cleaves several neuronal proteins associated with $\mathrm{AD}$, such as $\mathrm{APP}, \mathrm{tau}^{22}$ and presenilin 1 and $2,{ }^{23}$ Casp 6 also cleaves crucial cytoskeleton proteins including $\alpha$-Tub, and $\alpha$-actin-regulating post-synaptic density proteins, drebrin, spinophillin, $\alpha$-actinin- 1 and $-4 .^{4}$ Therefore, the activation of Casp6 may alter the neuronal cytoskeleton and be responsible for the abundant levels of dystrophic neurites present in AD brains.

Dystrophic neurites containing severely altered cytoskeletal organizations are a primary feature of $A D$ pathology. Often characterized by abnormal tau and phosphorylated tau immunoreactivity, dystrophic neurites in $A D$ show axonal swellings, varicosities, densely packed organelles, membranous dense bodies and vesicular profiles. ${ }^{24,25}$ Many of these features are present in the brain of aged APP Sw mutant transgenic mice. ${ }^{26}$ Overexpression of wild-type and mutant APP associated with familial AD induce axonal transport defects that result in organelle accumulation. ${ }^{26-31}$ Neuronal degeneration in APP wild-type transgenic mice brains is also associated with memory impairment. ${ }^{32}$ Surprisingly, in some animal models, APP-mediated axonopathies are independent of $\mathrm{A} \beta .^{26,33}$

As Casp6 is predominantly activated in sporadic and familial AD brains, in this study, we investigated if APP wild-type or familial AD-associated APP mutants induced Casp6 activity.

\section{Results}

Wild-type APP $_{695}\left(\right.$ APP $\left.^{W T}\right)$, Sw mutant APP $695\left(\right.$ APP $\left.^{\text {Sw }}\right)$ or the Lo mutant APP 695 (APP $\left.{ }^{L O}\right)$ induce Casp6-dependent neuritic enhanced green fluorescent protein (EGFP)positive beading in transfected primary human neurons. To assess whether familial AD-associated mutants activate Casp6 in terminally differentiated human neurons, primary cultures were transfected with pBudEGFP/ APP $^{W T}, /$ APP $^{S w}$ or $/ A P P^{\mathrm{Lo}}$ cDNA constructs. The APP and EGFP are independently expressed from the human elongation factor $1-\alpha$ and the cytomegalovirus (CMV) promoters, respectively. EGFP, thus, serves as a marker of transfection efficiency. The APP ${ }^{W T}$ represents overexpression of APP associated with trisomy 21 and a few FAD families. ${ }^{6}$ The expression of APP from these constructs was confirmed in Neuro-2a (N2a) cells, which in contrast to human neurons can be transfected with high efficiency for biochemical analyses (Supplementary Figure 1). Only $2 \mu \mathrm{g}$ of total proteins from the transfected cells were loaded on the western blot to avoid endogenous APP detection. Under these conditions, robust expression of APP was detected from each of the APP-expressing constructs, whereas none was observed in EGFP alone or mock-transfected cells; thus confirming APP expression under the CMV promoter of the pBud bigenic vector. As the CMV promoter is active in human primary neurons, these constructs were delivered with a biolistic particle delivery system, the most efficient transfection approach, as human neurons are not amenable to general viral infection systems or overly sensitive to liposomemediated transfections. In the absence of APP co-expression, the EGFP was homogeneously distributed in the cell body and neurites of transfected human primary neurons (Figure 1a and 1a inset). When APPWT, $A P P^{S w}$ or $\mathrm{APP}^{\mathrm{LO}}$ were co-expressed with EGFP, EGFP was often detected in an enlarged cell soma and appeared as beads-ona-string within the neurites (Figure $1 b-d$ ). This effect of APP on the EGFP distribution confirms the expected expression of APP from the pBudEGFP/APP constructs. Morphologically, EGFP-positive beads occurred at stochastic intervals and in varying sizes $(1-2.5 \mu \mathrm{m})$ along the neurites (Figure 1c inset). Nuclear staining with Hoechst 33342 demonstrated that the larger EGFP-positive agglomerates overlapped with nuclei, whereas the smaller beads did not (Figure 1b-d). Under phase contrast, neuritic EGFP-positive beads overlapped with a slight enlargement and darkened area of the neurite resembling varicosities, in the absence of compromised membrane integrity (Figure 1e).

Quantitative analyses demonstrated that 40-60\% APP ${ }^{W T}$-, $\mathrm{APP}^{\mathrm{Sw}}$ - or APP ${ }^{\mathrm{Lo}}$-transfected neurons developed an EGFPpositive beaded morphology by 48 to $72 \mathrm{~h}$ post transfection (Figure 1f). In contrast, EGFP expression in the absence of APP co-expression showed $<10 \%$ neuronal beading between 24 to $72 \mathrm{~h}$ of transfection. Together, these results indicate that APP ${ }^{W T}$, APP $^{\mathrm{Sw}}$ or APP ${ }^{\mathrm{Lo}}$ expression cause an agglomeration of EGFP within neurites of human neurons.

To investigate whether Casp6 activation was involved in neuritic EGFP-positive beading, APP ${ }^{W T}$-, APP ${ }^{\mathrm{Sw}_{-}}$or $\mathrm{APP}^{\mathrm{Lo}}$ transfected neurons were treated with $5 \mu \mathrm{M}$ of the Casp6 peptide inhibitor, z-VEID-fmk. Treatment with the Casp6 inhibitor significantly decreased EGFP-positive beading morphology in $\mathrm{APP}^{\mathrm{WT}}$-, $\mathrm{APP}^{\mathrm{Sw}}$ - or $\mathrm{APP}{ }^{\mathrm{Lo}}$-transfected neurons for up to $72 \mathrm{~h}$ (Figure 1f). Casp6 has recently been shown to be associated with neurodegeneration by causing beading of Tub in mouse neurons. ${ }^{21}$ We confirmed Casp6dependent Tub beading in human neurons (Supplementary Figure 2). Therefore, these results indicate that APP ${ }^{\mathrm{WT}}$, $\mathrm{APP}^{\mathrm{Sw}}$ or APP ${ }^{\mathrm{Lo}}$ overexpression cause Casp6-dependent neuritic degeneration.

Because peptide inhibitors of Casps can be promiscuous, we confirmed the involvement of Casp6 activity in APP- 
a
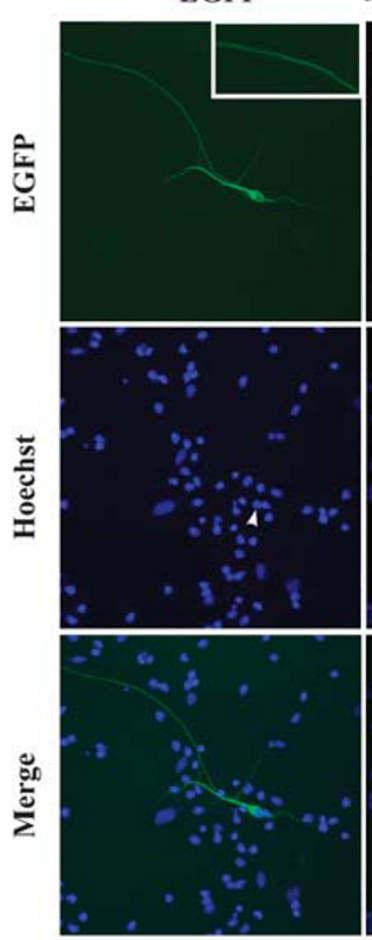

e

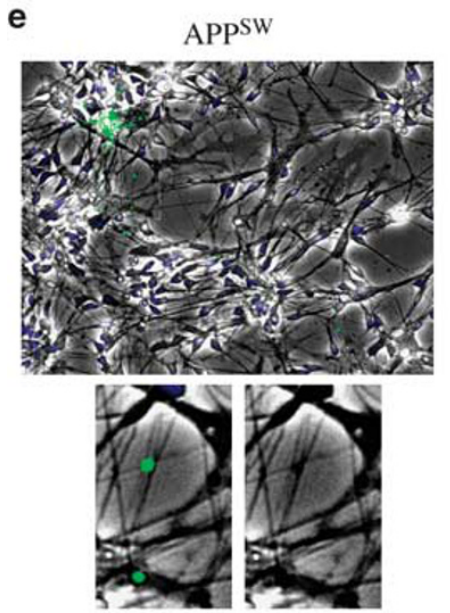

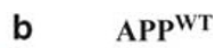
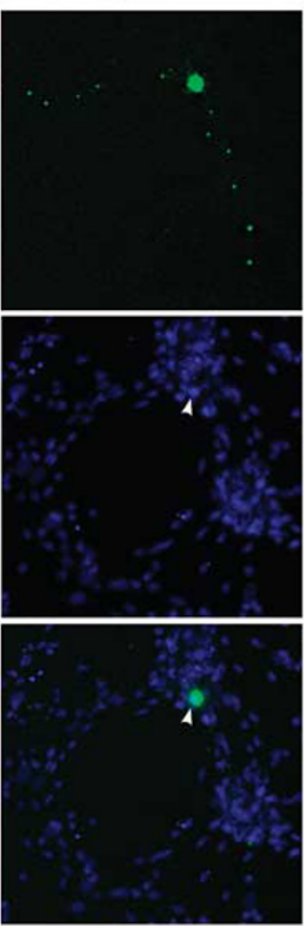

c
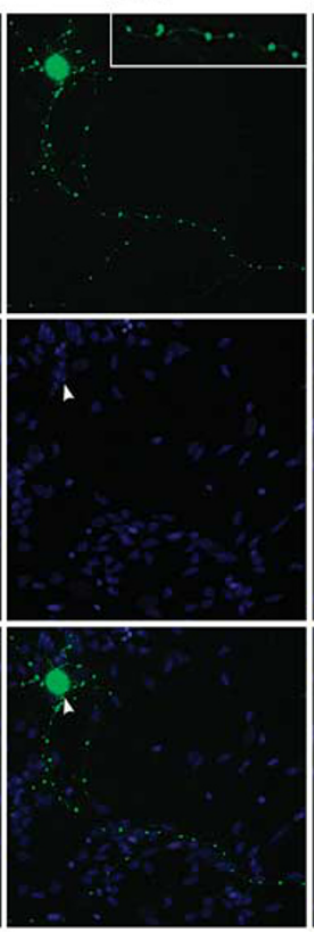

d $\quad \mathrm{APP}^{\mathrm{Lo}}$

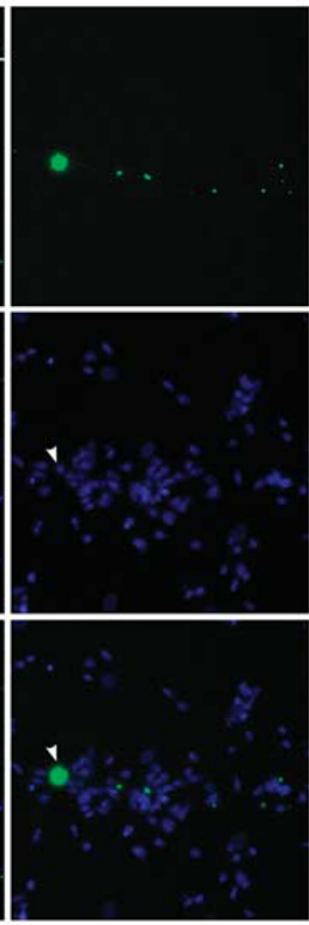

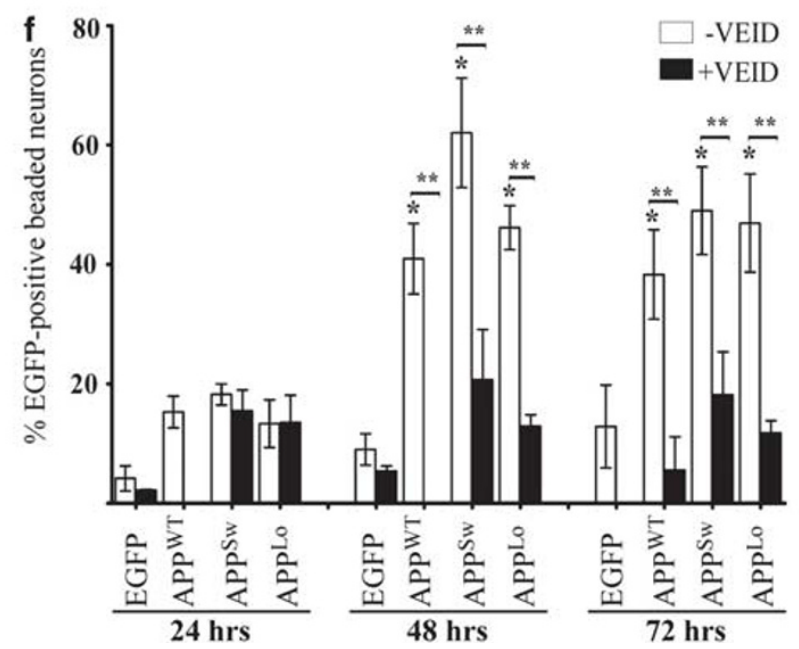

Figure 1 APP ${ }^{W T}, A P^{S w}$ or APPLo induce Casp6-dependent EGFP-positive neuritic beading in primary human neurons. (a-d) Fluorescence micrographs showing EGFP, Hoechst DNA stain and a merge of EGFP and Hoechst in (a) pBudEGFP- (b) pBudEGFP/APPWT-, (c) pBudEGFP/APPSw. and (d) pBudEGFP/APPLo ${ }_{\text {-transfected human }}$ neurons at $48 \mathrm{~h}$ post transfection. Insets given in panel $\mathbf{a}$ and $\mathbf{b}$ represent a higher magnification of the EGFP-positive neurites. Each picture was taken and enlarged at the same magnification. Arrows indicate the cell body of the transfected neuron. (e) Overlay of phase contrast, EGFP fluorescence and Hoescht-stained neuron depicted in Figure 1c. The lower panels represent a side-by-side comparison of the EGFP-positive beads with the phase contrast image. (f) Percentage of EGFP-positive beaded neurons relative to all EGFP-positive neurons incubated without (-VEID) or with $5 \mu \mathrm{M} z$-VEID-fmk (+VEID) for 24,48 or $72 \mathrm{~h}$. Data represents mean of three independent experiments \pm S.E.M. Each experiment represents 50-150 EGFP-positive neurons. *Represents a statistically significant difference $(P<0.01)$ between EGFP and APP versus EGFP only transfected neurons (paired for - VEID or + VEID). ${ }^{*}$ Represents a statistically significant difference of $P<0.001$ between - VEID and + VEID treatments

mediated neuritic beading with the catalytically mutated form of proCasp6, proCasp6C163A. The dominant-negative (DN) nature of proCasp6C163A was first tested on the self-activated form of Casp6 lacking its pro-domain (p20p10Casp6) in the human colon carcinoma HCT116 cell line. In these, the fluorogenic assay revealed that expression of proCasp6C163A completely abrogated p20p10Casp6- mediated VEIDase activity (Figure 2a). Western blot analyses of the transfected cells showed that proCasp6C163A was expressed as a $32 \mathrm{kDa}$ protein, whereas p20p10Casp6 migrated at $28 \mathrm{kDa}$, as expected (Figure $2 \mathrm{~b}$ ). Expression of p20p10Casp6 generated the p20 and p10 subunits of the active Casp6, consistent with the activity detected in Figure 2a. Co-expression of the proCasp6C163A with 

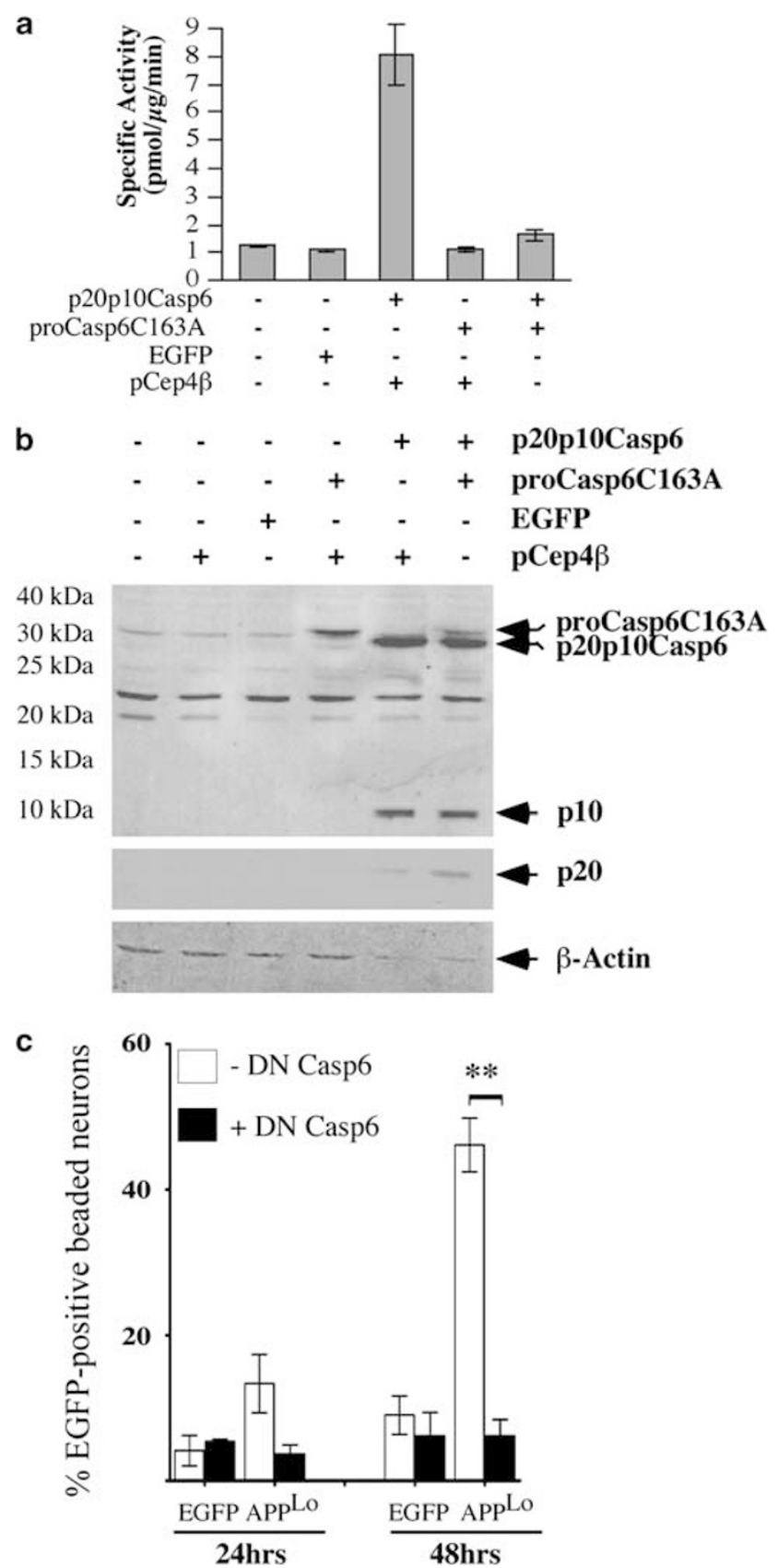

Figure 2 DN form of Casp6 inhibits APP ${ }^{L O}$-mediated EGFP-positive beading in primary human neurons. (a) Specific VEIDase activity and (b) western blot analysis with anti-p10Casp6 (top panel), 10630 neoepitope antiserum against p20 (middle panel) and anti- $\beta$-actin (bottom panel) in transfected HCT116 cellular protein extracts. (c) Percentage of EGFP-positive beaded neurons in cultures transfected with pBudEGFP or pBudEGFP/APP ${ }^{\text {Lo }}$ with pCep4 $\beta$ vector control (-DN Casp6) or pCep4 $\beta$-DN Casp6 ( + DN Casp6) at 24 and $48 \mathrm{~h}$ post transfection. Data represents mean \pm S.E.M. of three independent experiments. ${ }^{* *}$ Represents a statistically significant difference between -DN Casp6 and + DN Casp6 $(P<0.05)$

p20p10Casp6 reduced the level of the proCasp6C163A protein and increased the level of the p20 subunit indicating that proCasp6C163A was slightly processed by the active Casp6, and did not inhibit processing of the p20p10Casp6. These results are consistent with the hypothesis that DN inhibitors inhibit enzymatic Casp activity by generating a hetero-tetrameric enzyme containing catalytically inactive p20 subunits of the cognate Casp, and established the DN nature of proCasp6C163A (DN Casp6). Co-delivery of DN Casp6 with the pBudEGFP/APP ${ }^{\text {Lo }}$ in human neurons significantly prevented EGFP-positive neuritic beading, thus confirming the involvement of Casp6 activity in APP-mediated neuritic beading (Figure 2c).

APP $^{W^{T}}$-, APP ${ }^{\mathrm{Sw}}$ - or APP ${ }^{\mathrm{Lo}}$-induced EGFP-positive beads also contain tau and ubiquitin (Ub) proteins. To assess whether beading was specific to EGFP, red fluorescent protein (RFP)-tau was transfected alone or co-transfected with pBudEGFP, or with pBudEGFP/APPWT, /APP ${ }^{\mathrm{Sw}}$ or /APP ${ }^{\text {Lo }}$. RFP-tau was expressed and localized in the cell soma and neurites of neurons (Figure 3a). In RFP-tau and EGFP co-transfected neurons, both proteins showed an overlapping homogenous distribution in the neurons. In RFPtau neurons co-transfected with pBudEGFP/APP ${ }^{W T}$, /APP ${ }^{\text {SW }}$ or /APP ${ }^{\text {Lo }}$, EGFP- and RFP-positive beads appeared.

As the accumulation of $\mathrm{Ub}$ inclusions is a common feature of degenerating neurites, RFP-Ub was also co-transfected with EGFP and APP ${ }^{\text {Lo }}$ in neurons. By itself, RFP-Ub evenly distributed in the cell soma and in neurites (Figure $3 \mathrm{~b}$ ). However, co-transfection of RFP-Ub with EGFP and APP ${ }^{\text {Lo }}$ also caused RFP-Ub to accumulate in EGFP-positive beads. Together, the results indicate that the induced neuritic beading was not exclusive to EGFP, as it occurred with RFP-tau and RFP-Ub.

Neuritic beading is independent of $A \beta$ production in APP-transfected neurons. To determine whether neuritic beading may be a consequence of $\mathrm{A} \beta$ overproduction in APP-transfected neurons, we transfected human neurons with the $\mathrm{APP}^{\mathrm{MV}}$ construct, in which the methionine 596 was mutated to a valine to prevent processing by $\beta$-secretase and generation of $\mathrm{A} \beta$, as previously demonstrated. ${ }^{34}$ The APP ${ }^{\mathrm{MV}}$ protein was expressed from the pBudEGFP/APP ${ }^{M V}$ construct at levels similar to those of the APP ${ }^{S W}$ protein (Supplementary Figure 1). In human neurons, pBudEGFP/ $\mathrm{APP}^{\mathrm{MV}}$ induced EGFP-positive beads at a level consistently observed in pBudEGFP/APP ${ }^{\mathrm{WT}}$-transfected neurons (Figure 4a). Similarly, the presence of the Lo mutation in $\mathrm{APP}^{\mathrm{MV}}$ also generated $30.05 \pm 6.15 \%$ beading $(n=444$ transfected cells), indicating that the $\mathrm{APP}^{\mathrm{MV}}$-mediated beading was not synergistic with the APP ${ }^{\text {Lo }}$-mediated beading. z-VEID-fmk significantly inhibited $A P P^{M V}$-induced EGFP-positive beading, indicating that EGFP-positive beading was dependent on Casp6 activity (Figure 4b). We have previously reported that low levels of $A \beta_{1-42}$ or $A \beta_{1-40}$ induce neuritic beading of human neurons after $72 \mathrm{~h}$ of treatment and also render neurons vulnerable to a secondary insult. ${ }^{35}$ To assess if $\mathrm{A} \beta_{1-42}$ may function synergistically with $\mathrm{A} \beta$-independent neuritic beading in pBudEGFP/APP ${ }^{\mathrm{MV}}$ transfected neurons, these were treated with $50 \mathrm{nM} \mathrm{A} \beta_{1-42}$ or control reverse peptide $\mathrm{A} \beta_{42-1}$. $\mathrm{A} \beta_{1-42}$ increased levels of beading in pBudEGFP/APP ${ }^{\mathrm{MV}}$-transfected neurons after $24 \mathrm{~h}$ of incubation, but the increase did not reach statistical significance compared with $\mathrm{A} \beta_{42-1}$ (Figure $4 \mathrm{c}$ ). However, beading in $\mathrm{APP}^{\mathrm{MV}}$-transfected cells treated with $\mathrm{A} \beta_{1-42}$ was significantly inhibited by DN Casp6. These results confirmed 
a
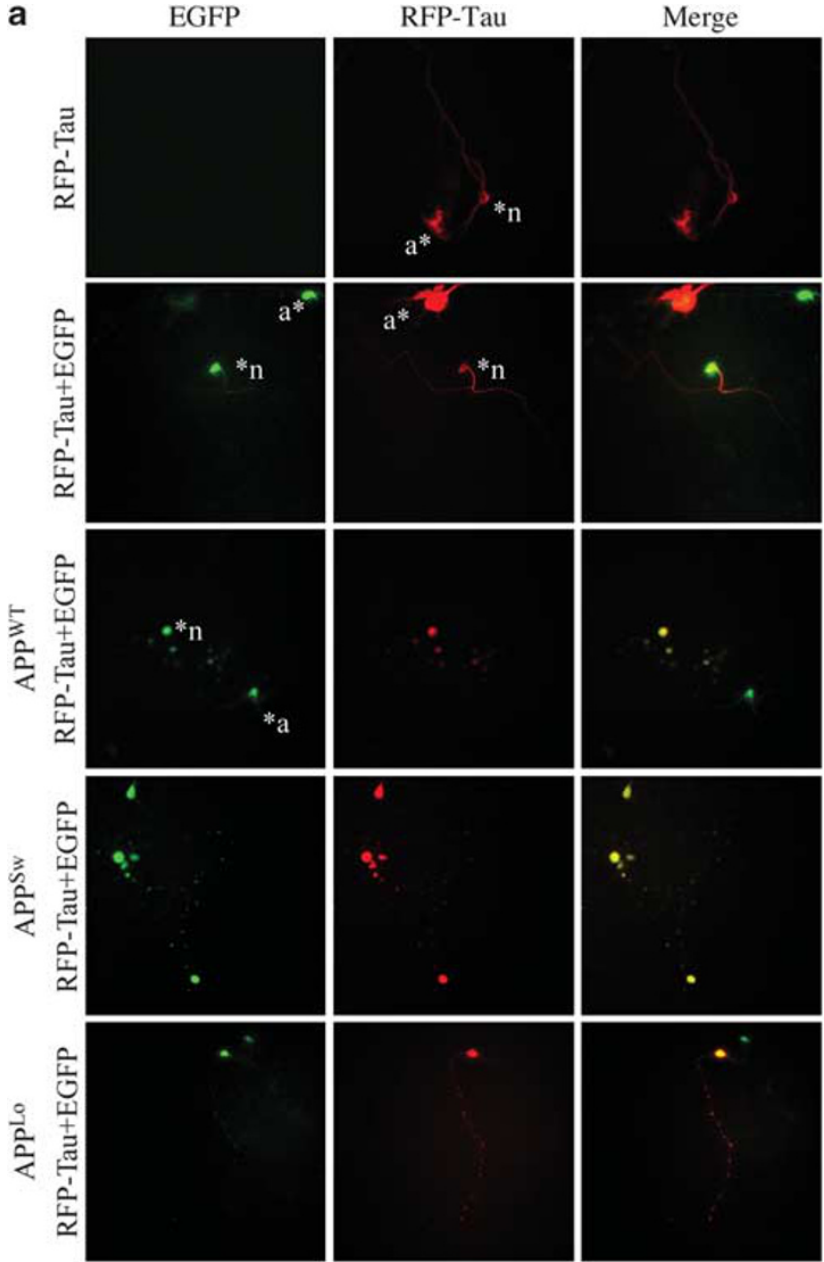

b

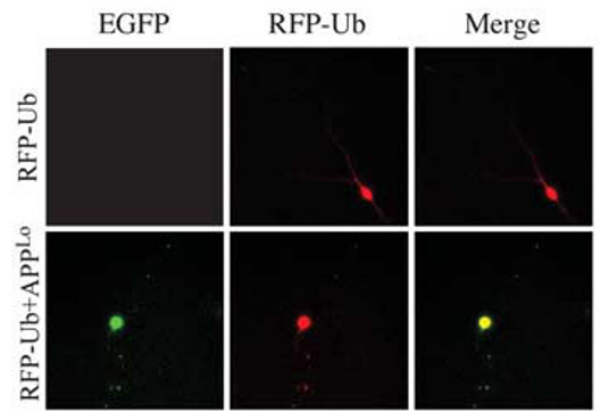

Figure 3 APP-induced neuritic beads contain tau and Ub proteins in addition to EGFP. Fluorescence micrographs of EGFP, RFP or a merged image of EGFP and RFP in human neurons transfected with (a) RFP-tau only, RFP-tau and pBudEGFP, or RFP-tau and pBudEGFP/APP ${ }^{W T}$, pBudEGFP/APP ${ }^{\mathrm{Sw}}$, or pBudEGFP/APP $^{\mathrm{Lo}}$ or (b) RFP-Ub or RFP-Ub and pBudEGFP/APP ${ }^{\mathrm{L}}$. * $n$ indicates a transfected neuron, *a indicates a transfected astrocyte

that the beading was dependent on Casp6 activity, but was independent of $\mathrm{A} \beta$.

APP $^{W T}$, APP $^{\text {Sw }}$ or APP ${ }^{\text {Lo }}$ cause Casp6-dependent EGFPpositive cell death. To determine whether APP expression induces cell death, primary human neurons were transfected with pBudEGFP/APP ${ }^{\mathrm{WT}}$, $/ \mathrm{APP}^{\mathrm{Sw}}$ or $/ \mathrm{APP}^{\mathrm{Lo}}$, and the total number of EGFP-expressing neurons monitored live by fluorescence microscopy every $24 \mathrm{~h}$ for up to $72 \mathrm{~h}$. Results were expressed relative to the $24 \mathrm{~h}$ time point (Figure $5 \mathrm{~A}$ ). EGFP-expressing neurons were maintained up to $72 \mathrm{~h}$; however, EGFP-positive beading in pBudEGFP/APPWT-, pBudEGFP/APP ${ }^{\mathrm{Sw}}$ - and pBudEGFP/APP ${ }^{\text {Lo }}$-transfected neurons decreased by 50,44 and $36 \%$, respectively, within $48 \mathrm{~h}$ post-transfection. After $72 \mathrm{~h}$, only $20 \%$ of EGFP-positive neurons remained in pBudEGFP/APP-transfected neurons. Treatment with $5 \mu \mathrm{M}$ of z-VEID-fmk significantly prevented EGFP-positive neuronal dropout in neurons transfected with pBudEGFP/APP ${ }^{W T}$ or pBudEGFP/APP ${ }^{\text {Lo }}$ at the $48 \mathrm{~h}$ time point (Figure 5A). By $72 \mathrm{~h}$, z-VEID-fmk significantly attenuated neuronal dropout levels in all pBudEGFP/APPtransfected neurons. Furthermore, co-expression of the DN Casp6 with the pBudEGFP/APP ${ }^{\text {Lo }}$ inhibited EGFP-positive neuronal dropout (Figure $5 \mathrm{~B}$ ). To confirm that the loss of EGFP-positive neurons was due to cell death, we assessed condensed chromatin with the cell permeable dye Hoechst 33342 at $48 \mathrm{~h}$ post transfection. In all, 50, 51 and $39 \%$ of pBudEGFP/APP ${ }^{W T}$-, /APP ${ }^{\mathrm{Sw}}$ - or APP ${ }^{\mathrm{Lo}}$-transfected neurons, respectively, exhibited condensed chromatin that was completely inhibited by z-VEID-fmk (Figure 5C). Likewise, the condensed chromatin in pBudEGFP/APP ${ }^{\text {Lo }}$-transfected neurons was abolished by co-expression of DN Casp6 (Figure 5D). Additionally, transfected neurons counterstained with terminal deoxynucleotidyl transferase-mediated biotinylated UTP nick end labeling (TUNEL)-Red and Hoechst 33342 showed that beaded APP ${ }^{\text {Lo }}$-transfected neurons had both condensed chromatin and fragmented DNA (Figure 5E). Collectively, these results indicate that expression of APP ${ }^{W T}$, APP ${ }^{\mathrm{Sw}}$ or $\mathrm{APP}^{\mathrm{Lo}}$ cause Casp6dependent neuronal cell death.

Cell death is dependent on $A \beta$ in APP-transfected neurons. To determine whether cell death may be due to $\mathrm{A} \beta$, we measured condensed chromatin in pBudEGFP/ $A P P^{M V}$-transfected human neurons. In contrast to the pBudEGFP/APP ${ }^{\mathrm{WT}}$-transfected neurons, pBudEGFP/ $A P P^{M V}$-transfected human neurons did not show an increase in condensed chromatin after $48 \mathrm{~h}$ of transfection (Figure 6a). Similarly, the pBudEGFP/APP ${ }^{\mathrm{MV} / \mathrm{Lo}_{\text {-transfected }}}$ cells showed only $12.2 \pm 2.5 \%$ condensed chromatin ( $n=444$ transfected neurons). However, when treated with $\mathrm{A} \beta_{1-42}$, but not reverse peptide $\mathrm{A} \beta_{42-1}$, a significant increase in condensed chromatin was observed in the $A P P^{M V}$ transfected neurons (Figure 6b). Condensed chromatin was present in $30-70 \%$ of the beaded neurons but rare in nonbeaded neurons (Figure 6c). Furthermore, the DN Casp6 decreased $\mathrm{A} \beta_{1-42}$-mediated condensed chromatin in beaded neurons indicating that $A \beta$-mediated cell death in human neurons expressing $A P P^{M V}$ is also dependent on Casp6 activity (Figure $6 \mathrm{c}$ ). Together, these results indicate that the $\mathrm{A} \beta$ generated from APP overexpression in transfected neurons is responsible for cell death.

\section{Discussion}

In this study, we showed that conditions associated with familial $A D$, such as APP ${ }^{W T}$ overexpression or the expression of APP mutants, induce Casp6-dependent but $A \beta$-independent neuritic degeneration in primary cultures of human 

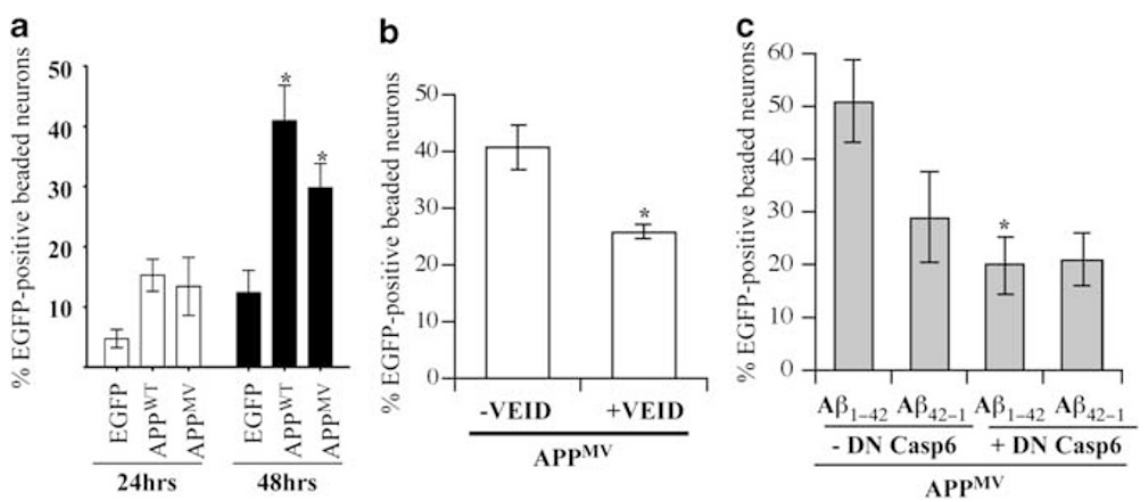

Figure 4 EGFP-positive beads in APP-transfected human neurons do not depend on A $\beta$ overproduction. (a) Percentage of EGFP-positive beaded neurons in pBudEGFP-, pBudEGFP/APPWT. or pBudEGFP/APPMV -transfected neurons after 24 and $48 \mathrm{~h}$. ${ }^{*}$ Represents a statistically significant difference between pBudEGFP- and pBudEGFP/ APP-transfected neurons $(P<0.05)$. (b) Percentage of EGFP-beaded neurons transfected with APPMV and cultured $48 \mathrm{~h}$ in the absence $(-V E I D)$ or presence $\left(+\right.$ VEID) of $5 \mu \mathrm{M} z$-VEID-fmk. ${ }^{*} P<0.02$. (c) Percentage of EGFP-positive beaded neurons transfected with pBudEGFP/APP ${ }^{\mathrm{MV}}$ and pCep4 $\beta$ (-DN Casp6) or pCep4 $\beta$ proCasp6C163A ( + DN Casp6) and cultured $24 \mathrm{~h}$ before treating for an additional $24 \mathrm{~h}$ with $50 \mathrm{nM} \mathrm{A} \beta_{1-42}$ or control reverse peptide $\mathrm{A} \beta_{42-1}$. ${ }^{*}$ Represents a $P<0.02$ difference between $A \beta_{1-42}-D N$ Casp6 and $A \beta_{1-42}+$ DN Casp6. No other statistically significant differences were obtained. For (a-c), data represents mean \pm S.E.M. of three independent experiments

neurons. Overexpression of APPWT, APPSw or APP ${ }^{L O}$ in human neurons resulted in the formation of neuritic swellings or varicosities, wherein proteins such as EGFP, RFP-tau and RFP-Ub accumulated rather than homogeneously distributed within the neurites. Inhibition of APP-mediated neuritic beading by the irreversible z-VEID-fmk peptide inhibitor and DN Casp6, confirmed the importance of Casp6 for neuritic beading. As overexpression of APP ${ }^{W T}$ or APP mutants result in increased levels of $A \beta,{ }^{11,36}$ it was logical to associate $A \beta$ with Casp6-dependent neuritic beading. However, in this study, we showed that $A \beta$ was not responsible. The APPMV mutated at the $\beta$-secretase site and thus unable to generate $\mathrm{A} \beta,{ }^{34}$ retained its ability to induce Casp6-dependent neuritic beading. Furthermore, the addition of physiologically high levels of $A \beta_{1-42}$ to the APPMV -transfected neurons did not enhance the level of neuritic beading. That neuritic disruption is distinct from $A \beta$ production is consistent with recently published results that showed that axonal defects are not dependent on A $\beta$ in an APP overexpression murine model. ${ }^{33}$ That Casp6 is responsible for neurodegeneration is entirely consistent with the recently emerging role for Casp6 in neuronal degeneration of mouse primary neurons. ${ }^{21}$ This data suggest that Casp6 may also be responsible for the previously reported axonopathy and transport deficits caused by APPWT, APP ${ }^{\mathrm{SW}}$ or APP ${ }^{\mathrm{LO}}$ overexpression in vitro and in vivo. ${ }^{26,27,29}$ Together with the present observations, the ability of Casp6 to cleave several important cytoskeletal proteins in human neurons ${ }^{4}$ and the strong activity of Casp 6 in neuropil threads, neuritic plaques and neurofibrillary tangles of sporadic and familial $A D$ neurons ${ }^{1-3}$ suggest that Casp6 has a predominant role in the neurodegenerative events of AD. These findings indicate that inhibition of Casp6 may be an efficient treatment against neurodegeneration in AD.

We also showed that APPWT overexpression or the expression of APP mutants induced Casp6- and A $\beta$ dependent cell death. Indeed, APPWT, APPSw or APPLo expression in primary human neurons caused neuronal dropout, condensed chromatin, and TUNEL staining. This cell death depended on Casp6 activity, occurred uniquely in neurons that presented neuritic beads, and was inhibited by z-VEID-fmk or the DN Casp6. In contrast to neuritic beading, cell death was caused by $\mathrm{A} \beta$, as it was absent in APPMV. transfected human neurons, and treatment with $50 \mathrm{nM}$ of extracellular $\mathrm{A} \beta_{1-42}$ re-established cell death in APP ${ }^{\mathrm{MV}}$ transfected beaded neurons. This is entirely consistent with a previous report showing that the APP M596I mutant, which cannot undergo $\beta$-secretase cleavage, increases the resistance of APP FAD mutant-expressing cells to apoptosis. ${ }^{37}$ Interestingly, in the absence of APP overexpression, concentrations of $100 \mathrm{nM}$ extracellular $\mathrm{A} \beta_{1-42}$ do not induce cell death but reduce the levels of $\mathrm{Bcl}-2$ while increasing the levels of Bax in human neurons, and renders these exposed neurons vulnerable to oxidative stress. ${ }^{35}$ These results show that neither APP overexpression nor extracellular A $\beta$ are sufficient to initiate cell death in human neurons but a combination of the two lowers the threshold of neuronal resistance to cell death. This phenomenon is consistent with numerous studies, in which APP ${ }^{\mathrm{Sw}}$ and APP ${ }^{\mathrm{LO}}$ induced cell death in COS-NK1, PC12, NT2N, rat and mouse primary cortical neurons, although the exact culprit was not identified in these cells. ${ }^{7,9,38,39}$ Although overexpression of APP and A $\beta$ causes neuritic beading in transgenic mice, it does not produce overwhelming levels of cell death. ${ }^{40}$ The ability of $\mathrm{A} \beta$ to induce cell death in APP-overexpressing neurons in culture but not in transgenic mice may be explained by differences in intrinsic species-specific cell death pathways or neuroprotective effects from supporting cells. In AD, there is an insignificant amount of neuronal loss except early in layer II of the entorhinal cortex and later in the temporal cortex of advanced cases of AD. ${ }^{41}$ However, neurodegeneration in the absence of evident cell death, is prominent early in $A D$, as is synaptic loss which correlates best with the level of dementia. ${ }^{22}$ Therefore, the toxicity of A $\beta$ on APP-transfected neurons may reflect a non-physiological event that occurs easily in cell cultures but not in AD brains. It is also important to note that Casp6 activity is not associated with acute cell death 

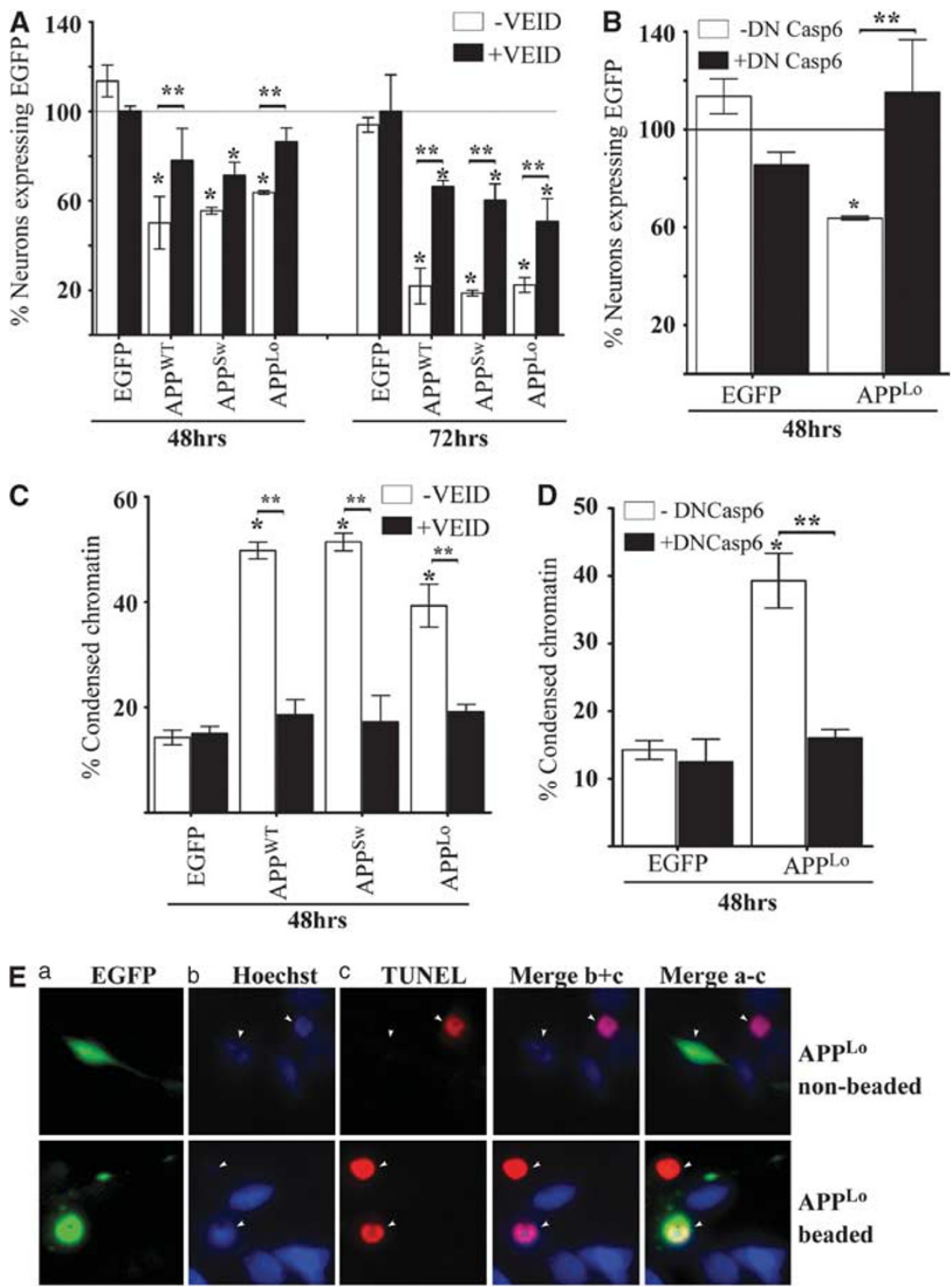

Figure $5 \mathrm{APP}^{\mathrm{WT}}, \mathrm{APP}^{\mathrm{Sw}}$ or APPLo induce Casp6-dependent EGFP-positive neuronal dropout and condensed chromatin. (A) Percentage of EGFP-positive neurons remaining after 48 and $72 \mathrm{~h}$ of transfection relative to those measured at $24 \mathrm{~h}$ of transfection (normalized at $100 \%$, solid line). Transfected neurons were incubated in the absence $(-\mathrm{VEID})$ or presence ( + VEID) of $5 \mu \mathrm{M}$ z-VEID-fmk. Data represents mean \pm S.E.M. of three independent experiments. ${ }^{*}$ Represents a $P<0.01$ statistically significant difference between pBudEGFP and pBudEGFP/APP-transfected neurons. ${ }^{* \star}$ Represents a statistically significant difference between -VEID and + VEID $(P<0.05)$. (B) Percentage of EGFP-positive neurons after $48 \mathrm{~h}$ of pBudEGFP or pBudEGFP /APP ${ }^{L 0}$ and DN Casp6 co-transfection. Data represents mean \pm S.E.M. of three independent experiments. ${ }^{*}$ Represents a $P<0.001$ statistically significant difference between -DN Casp6 and + DN Casp6. (C) Percentage of pBudEGFP, pBudEGFP/ $A P P^{W T}$, /APPSW or /APP ${ }^{\mathrm{LO}}$-transfected neurons treated with or without $5 \mu \mathrm{M} z$-VEID-fmk showing condensed chromatin after $48 \mathrm{~h}$ post transfection. Data represents the mean \pm S.E.M. of three independent experiments ( ${ }^{*}$ and ${ }^{* *}$ as in A). (D) Percentage of condensed chromatin-positive neurons co-transfected with pBudEGFP or pBudEGFP/ $A P{ }^{L 0}$ and DN Casp6 after $48 \mathrm{~h}$ post transfection. Data represents mean \pm S.E.M. of three independent experiments. ( ${ }^{*}$ and ${ }^{* *}$ as in B). (E) EGFP-positive pBudEGFP/APP ${ }^{\text {Lo }}$ transfected neuron stained with Hoechst and TUNEL

as observed with the other two effector Casps, Casp3 and Casp7. ${ }^{15,20}$ Similarly in $A D$, the active Casp6 is present in neurons that do not show apoptotic morphology. ${ }^{3}$ Furthermore, Casp6 activity causes neuritic degeneration but not cell death in primary mouse neurons. ${ }^{21}$ Therefore, the intense Casp6 activity observed in neuropil threads, neurofibrillary tangles and neuritic plaques is more likely associated with neurodegeneration than with cell death in AD. From these results, we conclude that removing $\mathrm{A} \beta$ without inhibiting Casp6 may have little effect in preventing the progressive dementia associated with sporadic or familial $A D$.

\section{Materials and Methods}

Cell culture. Primary human fetal neurons obtained with ethical approval from the McGill University Institutional Review Board were cultured as described previously. ${ }^{36}$. Briefly, 12-week-old fetal cerebrum were dissociated with trypsin, 

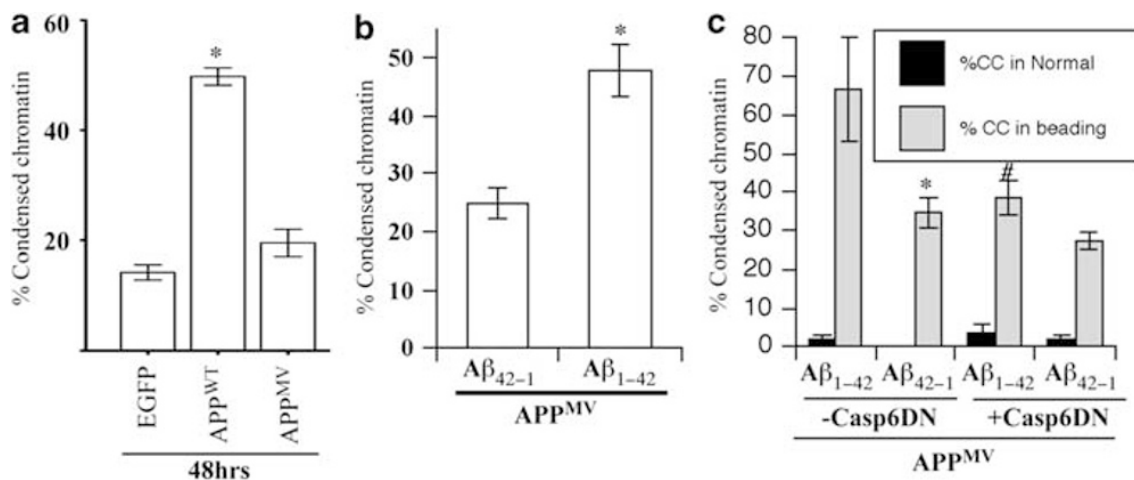

Figure 6 APP induces A $\beta$-dependent cell death. Percentage of condensed chromatin (\% CC) EGFP-positive neurons transfected with (a) pBudEGFP, pBudEGFP/APPWT or pBudEGFP/APPMV for $48 \mathrm{~h}$, (b) pBudEGFP/APP ${ }^{M V}$ for $24 \mathrm{~h}$ and treated with $50 \mathrm{nM} \mathrm{A} \beta_{1-42}$ or $50 \mathrm{nM} \mathrm{A} \beta_{42-1}$ for another $24 \mathrm{~h}$. *Represents a statistically significant difference of $P<0.05$ from pBudEGFP-transfected neurons. (c) Percentage of condensed chromatin EGFP-positive neurons co-transfected with pBudEGFP/APPMV and pCep4 $\beta$ vector (-DN Casp6) or pCep4 $\beta$-proCasp6C163A ( + DN Casp6) and cultured $24 \mathrm{~h}$ prior to additional $24 \mathrm{~h}$ treatment with $50 \mathrm{nM} \mathrm{A} \beta_{1-42}$ or control reverse peptide $\mathrm{A} \beta_{42-1}$. ${ }^{*}$ Represents $P<0.05$ difference between $\mathrm{A} \beta_{1-42}$ and $\mathrm{A} \beta_{42-1}$. " Represents a $P<0.05$ difference between -DN Casp6 and + DN Casp6

treated with deoxyribonuclease I, filtered through 130 and $70 \mu \mathrm{m}$ nylon mesh and plated on poly-lysine coated coverslips or dishes at a density of $3 \times 10^{6} \mathrm{cell} / \mathrm{s} / \mathrm{ml}$. Cultures generally contain $\sim 90 \%$ neurons and $10 \%$ astrocytes. Mouse neuroblastoma, N2a, cells were obtained from the American Type Culture Collection (Manassas, VA, USA) and maintained in MEM (Gibco-Invitrogen, Carlsbad, CA, USA) containing $1.5 \mathrm{~g}$ sodium bicarbonate (Gibco-Invitrogen), $1 \mathrm{mM}$ sodium pyruvate (Gibco-Invitrogen) and $10 \%$ fetal bovine serum (HyClone-Thermo Scientific, Waltham, MA, USA). Human colon carcinoma (HCT116) cells were obtained from the American Type Culture Collection and cultured according to manufacturer's protocol.

Cloning strategy and site directed mutagenesis of human APP. Full-length $\mathrm{APP}_{695} \mathrm{CDNA}\left(\mathrm{APP}{ }^{\mathrm{WT}}\right.$ ) was obtained from Dr. Georges Levesque (Laval University, Quebec, Canada) and subcloned into the bigenic eukaryotic pBudEGFP vector-expressing EGFP under the human elongation factor $1 \alpha$-promoter. To mimic the state of duplicated APP in familial AD, APPWT cDNA was subcloned under the human CMV promoter using the Hindlll and Notl sites to generate pBudEGFP/APPWT. The KM595/596NL Sw mutation and the V642l Lo mutation were generated by QuikChange site directed mutagenesis (Stratagene, La Jolla, CA, USA) using 5' -GATCTCTGAAGTGAATCTGGATGCAGAATTCCG-3' and $5^{\prime}$-CATAGCGACAGTGATCATCATCACCTTGGTG- $3^{\prime}$ primers to generate pBudEGFP/APPSw and pBudEGFP/APP ${ }^{\mathrm{LO}}$ vectors, respectively. The histidinetagged DN Casp6 C163A mutant (DN Casp6) was cloned in the $\mathrm{pCep} 4 \beta$ vector. The $\beta$-secretase uncleavable APP ${ }^{\mathrm{MV}}$ mutant was a kind gift from Dr. Jannic Boehm (University of Montreal, Quebec, Canada) ${ }^{34}$ and was subcloned into the pBudEGFP vector Sall and Xbal restriction sites. The Lo mutation was inserted in the APPMV by Quikchange mutagenesis. The pDs-Red RFP-tau (RFP-tau) construct was a kind gift from Dr. Yasuo Ihara (University of Tokyo, Tokyo, Japan) ${ }^{42}$ and the pRFP-C1 monomeric Ub (RFP-Ub) construct was purchased from Addgene (Cambridge, MA, USA) ${ }^{43}$ All constructs were confirmed by sequencing at the McGill University and Genome Quebec Innovation Centre.

Transfections. Neurons were plated on poly-L-lysine $(20 \mu \mathrm{g} / \mathrm{ml}$; Sigma-Aldrich, St Louis, MO, USA) coated polychlorotrifluoroethylene Aclar coverslips (Honeywell Aclar Film; http://www51.honeywell.com/sm/aclar/) at a density of $3 \times 10^{6} \mathrm{cell} / \mathrm{s} / \mathrm{ml}$ and were transfected with the Helios gene gun (Bio-Rad, Mississauga, Ontario, Canada) at a 100 p.s.i. pressure pulse of helium. The cartridges used for transfection were made of $0.033 \mathrm{mg}$ of DNA bound to $4.2 \mathrm{mg}$ of gold microcarrier beads prepared in $1 \mathrm{M}$ calcium chloride and $0.1 \mathrm{ml}$ of $0.05 \mathrm{M}$ spermidine. Approximately $1 \mu \mathrm{g}$ of DNA coated gold beads/cartridge was delivered to three coverslips of neurons per treatment. When transfecting cells with more than one construct, a ratio of 2:1 of RFP-tau or RFP-Ub to pBudEGFP, DN Casp6 and $\mathrm{APP}^{\mathrm{MV}}$ or $3: 1$ of DN Casp6 to APPLo was used to prepare cartridges. EGFP. positive cells were counted versus the total number of cells stained with Hoechst 33342 (Sigma-Aldrich) to estimate the transfection efficiency, which was around $0.001 \%$. Primary human neurons resist infection by commonly used viral vectors and lipid-based methods, and therefore, these methods could not be used for these studies. HCT116 cells were plated overnight in a six-well plate at a density of 400000 cells per well in McCoy's media (Gibco-Invitrogen) supplemented with $10 \%$ fetal bovine serum (Fisher, Ottawa, ON, Canada). Transfections were performed on the following day using polyethylenimine (Polysciences Inc., Warrington, PA, USA) at a $2 \mu \mathrm{g}$ concentration for every $1 \mu \mathrm{g}$ of DNA. The transfection complex was prepared in $100 \mu \mathrm{l}$ of serum-free media. At $6 \mathrm{~h}$ following transfection, complete media was replenished and cells were incubated for an additional $18 \mathrm{~h}$ to allow for expression.

Treatment of $\mathrm{APP}^{\mathrm{MV}}$-transfected neurons with $\mathrm{A} \boldsymbol{\beta}$ peptides. Fibrillar $A \beta$ s were prepared as described previously. ${ }^{44}$ Cells were transfected as described above, incubated for $24 \mathrm{~h}$ and then treated with $50 \mathrm{nM} \mathrm{A} \beta_{1-42}$ or $\mathrm{A} \beta_{42-1}$ control reverse peptide (diluted from a $25 \mu \mathrm{M}$ stock solution in culture media) for $24 \mathrm{~h}$ before assessing beading and condensed chromatin.

Measurement of neuritic beading in human neurons. Neurons displaying beaded EGFP were measured as a percent of the total number of EGFPpositive neurons at each $24 \mathrm{~h}$ time point for up to $72 \mathrm{~h}$. Results were obtained by averaging neuronal counts for at least 50 EGFP-positive neurons per experiment in three independent neuronal preparations.

Cell death assays: neuronal dropout. The number of EGFP-positive $\mathrm{APP}^{\mathrm{WT}}, \mathrm{APP}^{\mathrm{Sw}}, \mathrm{APP}^{\mathrm{Lo}}, \mathrm{APP}^{\mathrm{MV}}$ or DN Casp6-transfected human neurons in culture was assessed live over $72 \mathrm{~h}$ under fluorescence microscopy. For this analysis, the total number of transfected fluorescent neurons counted at $24 \mathrm{~h}$ was standardized to $100 \%$ and subsequent neuronal counts at 48 and $72 \mathrm{~h}$ were expressed as a percentage of the total number of neurons counted at $24 \mathrm{~h}$. A minimum of 50 neurons per experiment in three independent neuronal preparations was assessed.

Condensed chromatin. For condensed chromatin, $1 \mu \mathrm{g} / \mathrm{ml}$ of Hoescht 33342 was added to media $20 \mathrm{~min}$ before the live assessment, cells were washed twice in PBS pH 7.5 and fresh media was re-applied. EGFP-positive neurons displaying condensed chromatin visualized with Hoescht 33342 were counted.

Tunel. For DNA fragmentation, human neurons were stained with TUNEL-Red (Roche, Laval, Quebec, Canada). Briefly, neurons were fixed for $20 \mathrm{~min}$ at room temperature in $4 \%$ paraformaldehyde and $4 \%$ sucrose, and then permeabilized in $0.1 \%$ Triton $X-100$ and $0.1 \%$ sodium citrate before the staining.

Immunocytochemistry. Primary human neurons in culture were serumdeprived from $24-26 \mathrm{~h}$ and fixed with $4 \%$ paraformaldehyde and $4 \%$ sucrose for $20 \mathrm{~min}$ at room temperature. The fixed cells were permeabilized with $0.1 \%$ Triton X-100 and $0.1 \%$ sodium citrate, and treated with $10 \%$ goat serum for $20 \mathrm{~min}$. Primary antibodies consisting of anti-Tub (T3526; Sigma-Aldrich, 1/100), anti-tau (R42 from Dr. Hemant Paudel, McGill U Montreal, Canada, 1/250) anti-microtubuleassociated protein-2 (monoclonal clone AP20 from Sigma-Aldrich, 1/150) and anti-neurofilament (culture media from hybridoma JJ29A1AC from Dr. D Bignami's 
aboratory, Harvard Medical School, Boston, MA, USA) were applied overnight at $4^{\circ} \mathrm{C}$ and then washed in PBS three times before applying Cy3 Goat anti-mouse IgG (Millipore, Nepean, Ontario, Canada; 1/500) or Alexa 488 goat anti-rabbit serum (Molecular Probes, Burlington, Ontario, Canada; 1/500) secondary antibodies. Detection was done with a Nikon Eclipse TE2000-U fluorescence microscope (Mississauga, ON, USA). Images were acquired with an Orca-ER CCD camera (Improvision, Guelph, ON, Canada), Openlab (Improvision) and Adobe Photoshop 6.0 (Ottawa, ON, Canada) softwares.

Casp6 activity. To assess Casp6 activity in APPWT, APP ${ }^{\mathrm{SW}}$, APP ${ }^{\mathrm{LO}}$ or APPMV transfected neurons, neuronal cultures were pre-treated with $5 \mu \mathrm{M}$ of $z$-VEID-fmk (Biomol, Plymouth meeting, PA, USA) for $2 \mathrm{~h}$ before the gene gun transfections. Media containing the Casp6 inhibitor was changed every $24 \mathrm{~h}$ during the live assessment until the $72 \mathrm{~h}$ time point. In addition, neurons were co-transfected with the DN Casp6 as described above. To assess Casp6 activity in HCT116 cells, cells were harvested in CHAPS buffer (50 mM HEPES, 0.1\% CHAPS, $0.1 \mathrm{mM}$ EDTA $1 \mathrm{mM}$ DTT) containing protease inhibitors and total protein was quantified by BCA assay (ThermoScientific, Rockford, IL, USA). For measurement of Casp6 activity, fluorogenic Casp activity assays were performed with Stennicke's buffer $(50 \mathrm{mM}$ HEPES, pH 7.4, $100 \mathrm{mM} \mathrm{NaCl}, 0.1 \%$ CHAPS, $10 \mathrm{mM}$ DTT, $1 \mathrm{mM}$ EDTA, 10\% sucrose) containing $10 \mu \mathrm{M}$ Ac-VEID-AFC (Sigma-Aldrich) at $37^{\circ} \mathrm{C}$ using a Bio-Rad Fluoromark fluorometer (Hercules, CA, USA) at an excitation wavelength of $390 \mathrm{mM}$ and emission wavelength of $538 \mathrm{nM}$. Measurements were read every $2 \mathrm{~min}$ for $1 \mathrm{~h}$, and released pmol of AFC was calculated from an AFC standard curve run in parallel. The reaction was performed in $50 \mu \mathrm{l}$ total volume containing $10 \mu \mathrm{g}$ of total protein lysate.

Western blot analyses of Casp6. Western blotting analysis was performed with $30 \mu \mathrm{g}$ of $\mathrm{HCT} 116$ total protein lysate prepared in Laemmli buffer and eletrophoresed on a $15 \%$ SDS-PAGE gel. The following antibodies were used for subsequent detection. Anti-Casp6 polyclonal antiserum from NeoMarkers (Fremont, CA, USA) recognized the full length and p20 subunit of Casp6. The anti-p10Casp6 (Pharmingen) monoclonal antibody recognized the p10 subunit and full-length proCasp6 or proCasp6C163A. The anti-p20Casp6 10630 neoepitope antibody detecting the cleaved p20 subunit of active Casp6 was made in the laboratory. The anti- $\beta$-actin monoclonal antibody was raised against the 16 amino acids of the $\mathrm{N}$-terminus of the protein.

Statistical evaluations. Statistical analysis was performed with a one or two way analysis of variance and Tukey, Scheffé or Bonferroni post hoc test using the Statview software (SAS Institute Inc., Cary, NC, USA) or Graphpad Prism software (La Jolla, CA, USA).

\section{Conflict of interest}

The authors declare no conflict of interest.

Acknowledgements. We thank Jennifer Hammond for technical assistance. We thank the Birth Defects Research Laboratory at the University of Washington, Seattle, WA, USA for providing conceptal tissue for academic research (NIH HD 000836). We also thank Dr George Levesque (Laval University, Quebec City, Quebec, Canada) for the full-length APP ${ }_{695} \mathrm{CDNA}$, Dr. Jannic Boehm (University of Montreal, Montreal, Quebec, Canada) for the APP ${ }^{\mathrm{MV}}$ mutant construct and Dr. Yasuo Ihara (University of Tokyo, Japan) for the RFP-tau construct. We gratefully acknowledge Dr. Julie Jodoin and Dalia Halawani from our laboratory for their critical reading and suggestions of the manuscript. This work was supported by the Canadian Institute for Health Research MOP 81146, a Fond de Recherche en Santé du Quebec National Scholar Award and a McGill University James McGill Professorship to ALB.

1. Albrecht S, Bogdanovic N, Ghetti B, Winblad B, LeBlanc AC. Caspase-6 activation in familial alzheimer disease brains carrying amyloid precursor protein or presenilin I or presenilin II mutations. J Neuropathol Exp Neurol 2009; 68: 1282-1293.

2. Albrecht S, Bourdeau M, Bennett D, Mufson EJ, Bhattachariee M, LeBlanc AC. Activation of caspase-6 in aging and mild cognitive impairment. Am J Pathol 2007; 170: 1200-1209.

3. Guo H, Albrecht S, Bourdeau M, Petzke T, Bergeron C, LeBlanc AC. Active caspase-6 and caspase-6-cleaved tau in neuropil threads, neuritic plaques, and neurofibrillary tangles of Alzheimer's disease. Am J Pathol 2004; 165: 523-531.
4. Klaiman G, Petzke TL, Hammond J, LeBlanc AC. Targets of caspase- 6 activity in human neurons and Alzheimer disease. Mol Cell Proteomics 2008; 7: 1541-1555

5. Bertram L, Tanzi RE. Thirty years of Alzheimer's disease genetics: the implications of systematic meta-analyses. Nat Rev Neurosci 2008; 9: 768-778.

6. Rovelet-Lecrux A, Hannequin D, Raux G, Le Meur N, Laquerriere A, Vital A et al. APP locus duplication causes autosomal dominant early-onset Alzheimer disease with cerebral amyloid angiopathy. Nat Genet 2006; 38: 24-26.

7. McPhie DL, Golde T, Eckman CB, Yager D, Brant JB, Neve RL. Beta-secretase cleavage of the amyloid precursor protein mediates neuronal apoptosis caused by familial Alzheimer's disease mutations. Brain Res Mol Brain Res 2001; 97: 103-113.

8. Giambarella U, Murayama Y, Ikezu T, Fujita T, Nishimoto I. Potential CRE suppression by familial Alzheimer's mutants of APP independent of adenylyl cyclase regulation. FEBS Lett 1997; 412: 97-101

9. Yamatsuji T, Okamoto T, Takeda S, Murayama Y, Tanaka N, Nishimoto I. Expression of V642 APP mutant causes cellular apoptosis as Alzheimer trait-linked phenotype. EMBO J 1996; 15: 498-509.

10. Eckert A, Steiner B, Marques C, Leutz S, Romig H, Haass C et al. Elevated vulnerability to oxidative stress-induced cell death and activation of caspase- 3 by the Swedish amyloid precursor protein mutation. J Neurosci Res 2001; 64: 183-192.

11. Selkoe DJ, Podlisny MB. Deciphering the genetic basis of Alzheimer's disease. Annu Rev Genomics Hum Genet 2002; 3: 67-99.

12. Gervais FG, Xu D, Robertson GS, Vaillancourt JP, Zhu Y, Huang J et al. Involvement of caspases in proteolytic cleavage of Alzheimer's amyloid-beta precursor protein and amyloidogenic A beta peptide formation. Cell 1999; 97: 395-406.

13. LeBlanc A, Liu H, Goodyer C, Bergeron C, Hammond J. Caspase-6 role in apoptosis of human neurons, amyloidogenesis, and Alzheimer's disease. J Biol Chem 1999; 274: 23426-23436.

14. Srinivasula SM, Fernandes-Alnemri T, Zangrilli J, Robertson N, Armstrong RC, Wang L et al. The Ced-3/interleukin 1beta converting enzyme-like homolog Mch6 and the lamincleaving enzyme Mch2alpha are substrates for the apoptotic mediator CPP32. J Biol Chem 1996; 271: 27099-27106.

15. Klaiman G, Champagne N, LeBlanc AC. Self-activation of caspase-6 in vitro and in vivo: caspase-6 activation does not induce cell death in HEK293T cells. Biochim Biophys Acta 2009; 1793: 592-601.

16. Kang JJ, Schaber MD, Srinivasula SM, Alnemri ES, Litwack G, Hall DJ et al. Cascades of mammalian caspase activation in the yeast Saccharomyces cerevisiae. J Biol Chem 1999; 274: 3189-3198

17. Allsopp TE, McLuckie J, Kerr LE, Macleod M, Sharkey J, Kelly JS. Caspase 6 activity initiates caspase 3 activation in cerebellar granule cell apoptosis. Cell Death Differ 2000; 7: 984-993.

18. Doostzadeh-Cizeron J, Yin S, Goodrich DW. Apoptosis induced by the nuclear death domain protein p84N5 is associated with caspase-6 and NF-kappa B activation. $J$ Biol Chem 2000; 275: 25336-25341.

19. Cowling V, Downward J. Caspase- 6 is the direct activator of caspase-8 in the cytochrome c-induced apoptosis pathway: absolute requirement for removal of caspase- 6 prodomain. Cell Death Differ 2002; 9: 1046-1056.

20. Zhang Y, Goodyer C, LeBlanc A. Selective and protracted apoptosis in human primary neurons microinjected with active caspase-3, -6, -7, and -8. J Neurosci 2000; 20: 8384-8389.

21. Nikolaev A, McLaughlin T, O'Leary DD, Tessier-Lavigne M. APP binds DR6 to trigger axon pruning and neuron death via distinct caspases. Nature 2009; 457: 981-989.

22. LeBlanc AC. The role of apoptotic pathways in Alzheimer's disease neurodegeneration and cell death. Curr Alzheimer Res 2005; 2: 389-402.

23. van de Craen M, de Jonghe C, van den Brande I, Declercq W, van Gassen G, van Criekinge $W$ et al. Identification of caspases that cleave presenilin-1 and presenilin-2. Five presenilin-1 (PS1) mutations do not alter the sensitivity of PS1 to caspases. FEBS Lett 1999; 445: 149-154.

24. Dickson DW. The pathogenesis of senile plaques. J Neuropathol Exp Neurol 1997; 56: 321-339.

25. Perry G, Kawai M, Tabaton M, Onorato M, Mulvihill P, Richey P et al. Neuropil threads of Alzheimer's disease show a marked alteration of the normal cytoskeleton. J Neurosci 1991; 11: 1748-1755.

26. Stokin GB, Goldstein LS. Axonal transport and Alzheimer's disease. Annu Rev Biochem 2006; 75: 607-627

27. Salehi A, Delcroix JD, Belichenko PV, Zhan K, Wu C, Valletta JS et al. Increased APP expression in a mouse model of Down's syndrome disrupts NGF transport and causes cholinergic neuron degeneration. Neuron 2006; 51: 29-42.

28. Wirths $\mathrm{O}$, Weis J, Kayed R, Saido TC, Bayer TA. Age-dependent axonal degeneration in an Alzheimer mouse model. Neurobiol Aging 2007; 28: 1689-1699.

29. Nishimura I, Uetsuki T, Dani SU, Ohsawa Y, Saito I, Okamura H et al. Degeneration in vivo of rat hippocampal neurons by wild-type Alzheimer amyloid precursor protein overexpressed by adenovirus-mediated gene transfer. J Neurosci 1998; 18: 2387-2398.

30. Gotz J, Ittner LM, Kins S. Do axonal defects in tau and amyloid precursor protein transgenic animals model axonopathy in Alzheimer's disease? J Neurochem 2006; 98: 993-1006.

31. Lazarov O, Morfini GA, Lee EB, Farah MH, Szodorai A, DeBoer SR et al. Axonal transport, amyloid precursor protein, kinesin-1, and the processing apparatus: revisited. J Neurosci 2005; 25: 2386-2395. 
32. Simon AM, Schiapparelli L, Salazar-Colocho P, Cuadrado-Tejedor M, Escribano L, Lopez de Maturana $\mathrm{R}$ et al. Overexpression of wild-type human APP in mice causes cognitive deficits and pathological features unrelated to Abeta levels. Neurobiol Dis 2009; 33: 369-378.

33. Stokin GB, Almenar-Queralt A, Gunawardena S, Rodrigues EM, Falzone T, Kim J et al. Amyloid precursor protein-induced axonopathies are independent of amyloid-beta peptides. Hum Mol Genet 2008; 17: 3474-3486.

34. Citron M, Teplow DB, Selkoe DJ. Generation of amyloid beta protein from its precursor is sequence specific. Neuron 1995; 14: 661-670.

35. Paradis E, Douillard H, Koutroumanis M, Goodyer C, LeBlanc A. Amyloid Bpeptide of Alzheimer's disease downregulates Bcl-2 and upregulates Bax expression in human neurons. J Neurosci 1996; 16: 7533-7539.

36. LeBlanc A. Increased production of $4 \mathrm{kDa}$ amyloid beta peptide in serum deprived human primary neuron cultures: possible involvement of apoptosis. J Neurosci 1995; 15 : 7837-7846.

37. Esposito L, Gan L, Yu GQ, Essrich C, Mucke L. Intracellularly generated amyloid-beta peptide counteracts the antiapoptotic function of its precursor protein and primes proapoptotic pathways for activation by other insults in neuroblastoma cells. J Neurochem 2004; 91: 1260-1274.

38. Luo JJ, Wallace W, Riccioni T, Ingram DK, Roth GS, Kusiak JW. Death of PC12 cells and hippocampal neurons induced by adenoviral-mediated FAD human amyloid precursor protein gene expression. J Neurosci Res 1999; 55: 629-642.

39. Zhao B, Chrest FJ, Horton Jr WE, Sisodia SS, Kusiak JW. Expression of mutant amyloid precursor proteins induces apoptosis in PC12 cells. J Neurosci Res 1997; 47: 253-263.
40. Small SA, Duff K. Linking Abeta and tau in late-onset Alzheimer's disease: a dual pathway hypothesis. Neuron 2008; 60: 534-542.

41. Gomez-Isla T, Hollister R, West H, Mui S, Growdon J, Petersen R et al. Neuronal loss correlated with but exceeds neurofibrillary tangles in Alzheimer's disease. Ann Neurol 1996; 41: 17-24.

42. Oyama F, Kotliarova S, Harada A, Ito M, Miyazaki H, Ueyama $Y$ et al. Gem GTPase and tau: morphological changes induced by gem GTPase in $\mathrm{CHO}$ cells are antagonized by tau. J Biol Chem 2004; 279: 27272-27277.

43. Bergink S, Severijnen LA, Wijgers N, Sugasawa K, Yousaf H, Kros JM et al. The DNA repair-ubiquitin-associated HR23 proteins are constituents of neuronal inclusions in specific neurodegenerative disorders without hampering DNA repair. Neurobiol Dis 2006; 23: 708-716.

44. Zhang Y, McLaughlin R, Goodyer C, LeBlanc A. Selective cytotoxicity of intracellular amyloid $\beta$ peptide $1-42$ through $p 53$ and Bax in cultured primary human neurons. $J$ Cell Biol 2002; 156: 519-529.

Cell Death and Disease is an open-access journal published by Nature Publishing Group. This work is licensed under the Creative Commons Attribution-Noncommercial-No Derivative Works 3.0 Unported License. To view a copy of this license, visit http://creativecommons.org/licenses/by-nc-nd/3.0/

Supplementary Information accompanies the paper on Cell Death and Disease website (http://www.nature.com/cddis) 